\title{
A method for extracting calibrated volatility information from the FIGAERO- HR-ToF-CIMS and its experimental application
}

\section{Thomas J. Bannan et al.}

Correspondence to: Thomas J. Bannan (thomas.bannan@manchester.ac.uk)

The copyright of individual parts of the supplement might differ from the CC BY 4.0 License. 
(Multipeak) FIGAERO thermograms can be evaluated with the GUFIT (Gothenburg University FItting for Thermograms) procedure. GUFIT is written in Python 3.6.0 using NumPy (v 1.11.3), SciPy (v 0.19.1), lmfit (0.9.6) and pandas (v 0.19.2) libraries. Thermogram data is loaded into Python and corrected for the flow difference between sampling on the filter (4LPM) and desorption from the filter (2LPM). $\mathrm{T}_{\text {Max }}$ guesses for the initial parameters are calculated by finding minima in

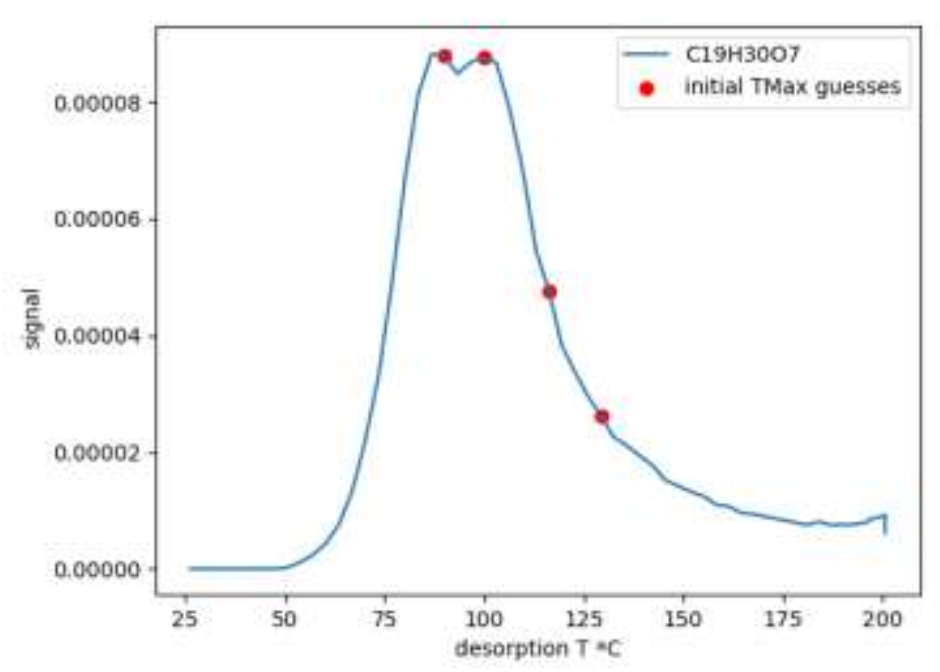

Figure S1. Initial guesses of $\mathrm{T}_{\mathrm{Max}}$ values for the thermogram of $\mathrm{C}_{19} \mathrm{H}_{30} \mathrm{O}_{7}$, a carboxylic acid dimer from limonene oxidation with ozone and $\mathrm{OH}$ under humid conditions, data from (Hammes et al., 2018). The blue line shows normalized high resolution data obtained with tofware (ARI tofware).

10 The exponentially modified Gaussian (EMG, (Eq.1)), a Gaussian function convoluted with an exponential decay function, is used as the single peak shape for the model (Jeansonne and Foley, 1991;Goodman and Brenna, 1994).

$f(x)=\frac{A}{2 \tau} \exp \left[\left(\frac{W}{2 \tau}\right)^{2}+\frac{T-x}{\tau}\right]\left[\operatorname{erf}\left(\frac{x-T}{\sqrt{2} W}-\frac{W}{\sqrt{2} \tau}\right)+1\right]$

Where $\mathrm{A}$ is the area, $\mathrm{T}$ is $\mathrm{T}_{\mathrm{Max}}, \mathrm{W}$ the width of the Gaussian, $\tau$ the exponential damping term and erf is the error function. The initial guesses were empirically determined for the 3 terms $\mathrm{A}, \tau$ and $\mathrm{W}$ were calculated from $\mathrm{T}_{\mathrm{Max}}$ and the signal intensity $\mathrm{H}$ at $\mathrm{T}_{\mathrm{Max}}$ :

$\mathrm{W}=\frac{\mathrm{T}_{\mathrm{Max}}}{2}$

$\tau=\mathrm{T}_{\mathrm{Max}}$

$\mathrm{A}=\frac{\mathrm{H} \sqrt{\mathrm{W}}}{0.3989}$

The following, empirically determined, constraints were set for the parameters for EMG 1, 2 and 3:

$20 \mathrm{~A} 1, \min =0.0001, \max =\mathrm{inf}$

$\mathrm{TMax} 1, \min =\mathrm{TMax} 1-10, \max =\mathrm{TMax} 1+3$

$\mathrm{W} 1, \min =0, \max =30$

$\tau 1, \min =0, \max =\inf$

A2, $\min =0.00001, \max =\inf$

$25 \mathrm{TMax} 2, \min =\mathrm{TMax} 2-3, \max =\mathrm{TMax} 2+20$

$\mathrm{W} 2, \min =5, \max =30$

$\tau 2, \min =0, \max =\inf$

A3, $\min =0$, max $=$ inf 
$\mathrm{TMax} 3, \min =\mathrm{TMax} 3, \max =\mathrm{TMax} 3+20$

W3, $\min =0, \max =$ inf

$\tau 3, \min =0, \max =\inf$

To accurately model the shape of a thermogram, two to three EMG were convoluted. The lmfit package for Python

5 (Newville et al., 2014) was used to fit the convoluted EMG model to real thermogram data by minimizing the residual function, example with three EMG in the following case:

res $=(E M G 1+E M G 2+E M G 3)-$ rawdata

The following fitting methods and smoothing parameters were compared to find the best fit of the Gaussian model to the data:

10 Fit methods: leastsq, nelder, powell

Window types for smoothing: flat, hanning, hamming, bartlett, blackman

Window lengths for smoothing: $1,3,5,7,9,11,13,15)$

The combination with the lowest residual was chosen as the final fit (see Figure S 2 and Figure S 3. If the sum of the residual was larger than $15 \%$ of the sum of peak 1 , the fitting process was repeated using the sciPy .curve_fit function. If the sciPy fit residual was also larger than $15 \%$ the lmfit results were exported. A flowchart illustrating the GUFIT algorithm can be seen in Figure S 4.

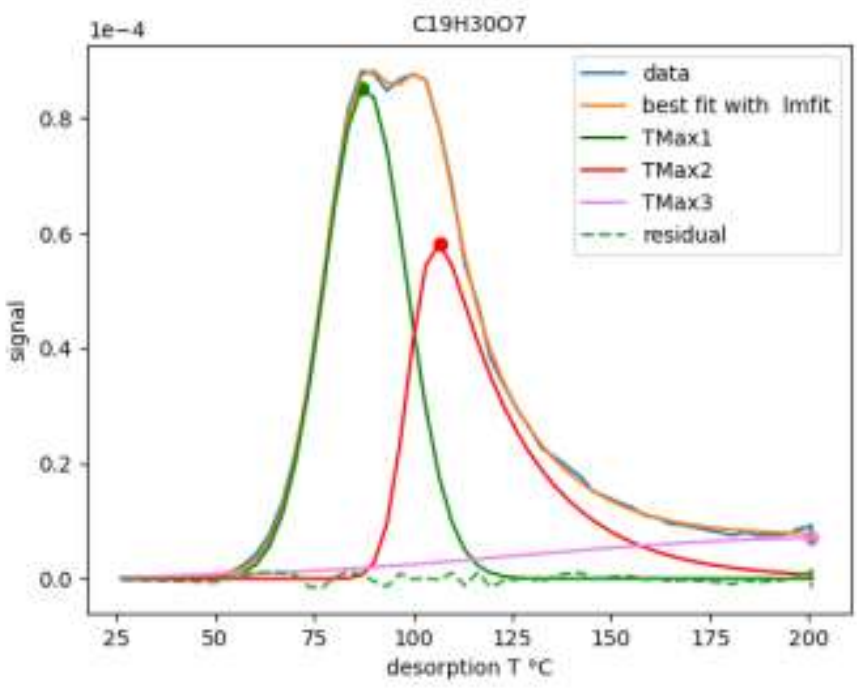

Figure S 2. Successful fit with lmfit for the carboxylic acid dimer $\mathrm{C}_{19} \mathrm{H}_{30} \mathrm{O}_{7}$. The orange curve represents the fitted model and the dashed green line is the residual between the raw data (blue) and the model. 


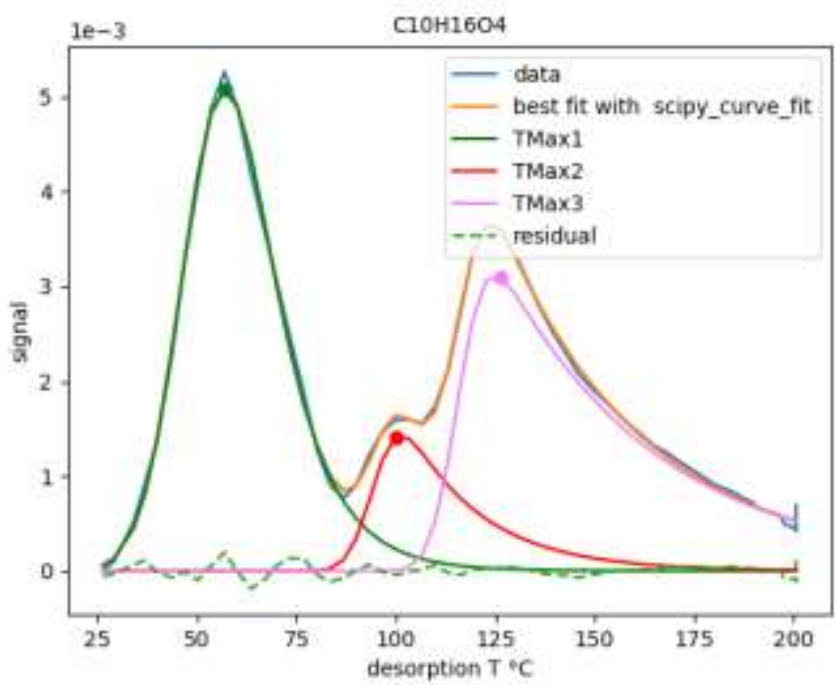

Figure S 3. Curve fit with SciPy for the carboxylic acid $\mathrm{C}_{10} \mathrm{H}_{16} \mathrm{O}_{4}$, data from (Hammes et al., 2018).

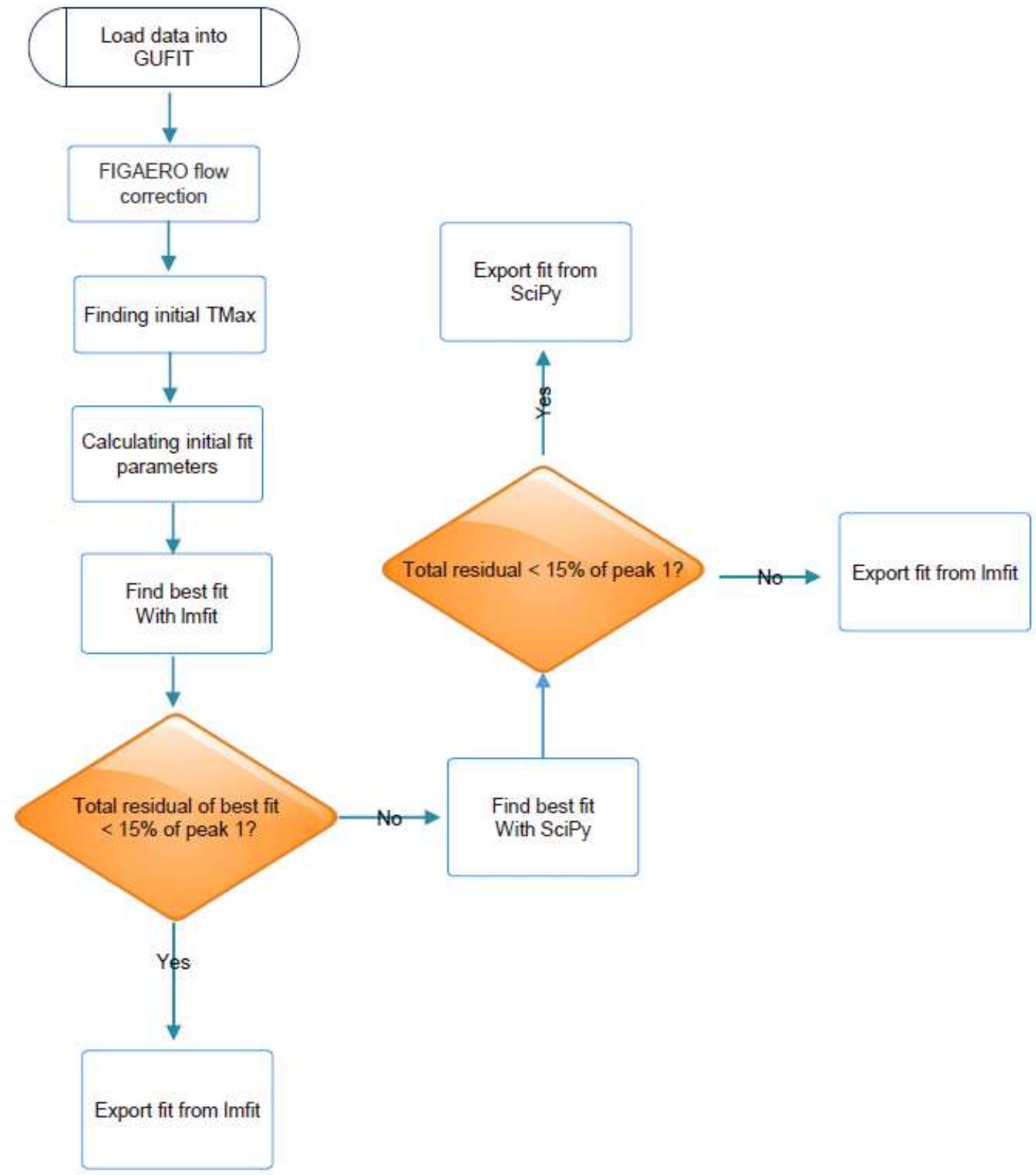

Figure S 4. Flowchart of the GUFIT algorithm. 


\section{References}

Goodman, K. J., and Brenna, J. T.: Curve fitting for restoration of accuracy for overlapping peaks in gas chromatography/combustion isotope ratio mass spectrometry, Analytical chemistry, 66, 1294-1301, 10.1021/ac00080a015, 1994.

Hammes, J., Lutz, A., Mentel, T., Faxon, C., and Hallquist, M.: Carboxylic acids from limonene oxidation by ozone and OH radicals: Insights into mechanisms derived using a FIGAERO-CIMS, Atmospheric Chemistry and Physics Discussions, 123, 10.5194/acp-2018-1004, 2018.

10 Jeansonne, M. S., and Foley, J. P.: Review of the Exponentially Modified Gaussian (Emg) Function since 1983, Journal of Chromatographic Science, 29, 258-266, DOI 10.1093/chromsci/29.6.258, 1991.

Newville, M., Stensitzki, T., Allen, D. B., and Ingargiola, A.: LMFIT: Non-Linear Least-Square Minimization and CurveFitting for PythonII, in, @zenodo_org, 2014. 